\title{
Analysis of risk factors, clinical features and visual outcome in cases of non-arteritic anterior ischemic optic neuropathy (NA-AION) at a tertiary eye care centre in India
}

\author{
Aditya Vikram Sharma ${ }^{1, *}$, Aditi Sharma ${ }^{2}$ \\ ${ }^{1,2}$ Consultant, Dept. of Ophthalmology, Kalyani Eye Hospital, Rajasthan, India \\ *Corresponding Author: Aditya Vikram Sharma \\ Email: avsharma007@yahoo.com
}

\begin{abstract}
We did an analysis of risk factors, clinical features and visual outcome in cases of non-arteritic anterior ischemic optic neuropathy (NA-AION) at a tertiary eye care centre in India.

This is a prospective, observational, clinical study. A total of 60 eyes of 60 consecutive adult patients with disc oedema, due to non-arteritic AION, presenting in the Neuro-ophthalmology clinic of a tertiary care eye hospital, were enrolled over a period of 18 months (in specified period) according to the inclusion criteria.

The study, including patient recruitment and data collection, was approved by the Institutional Review Board. All patients provided written informed consent after they received information about the disease and its relevant investigations along with the risks associated.

Sample collection was done by application of inclusion and exclusion criteria on the patients presenting to the clinic. The study was carried out for a period of 6 months for each subject. The major outcomes of the study were:

Metabolic Syndromes are the most significantly associated risk factors for the occurrence of NA AION.

Clinical investigations mainly show the characteristic findings as mentioned in literature, with very little variation. However the importance of clinical examination, mainly pupillary reaction and fundus evaluation by slit lamp biomicroscopy, cannot be overlooked.

Visual acuity is highly variable at presentation with cases even presenting with normal or near normal visual acuity. Visual outcome showed significantly improvement within 6 months of presentation. Non arteritic anterior ischemic optic neuropathy (NAION) was the most common form of ischemic optic neuropathy in our study.

It is an idiopathic, ischemic insult of the optic nerve head characterized by acute, monocular, painless visual loss with optic disc swelling.

The systemic risk factor which we found to be most strongly associated with was diabetes mellitus followed by systemic hypertension. Also an ocular risk factor of small cup size. We have few inherent limitations in the study mainly with the occurrence of symptoms and time of presentation of the patient, which may have a bearing on the final outcome of the disease. Also a short follow up limited us regarding study of the stabilization of visual acuity over a longer duration.

However our study gives an overview of prevalent risk factors and clinical characteristics. It also shows a significant visual outcome after 6 months in NA-AION.
\end{abstract}

Keywords: Clinical features, NA AION, Visual outcome, India.

\section{Introduction}

Non-arteritic anterior ischemic optic neuropathy (NA-AION) is one of the common causes of sudden, painless loss of vision present on awakening from sleep. ${ }^{1}$

It is more prevalent in middle-aged and elderly Caucasian men and women. Hypertension, diabetes, and nocturnal hypotension are major risk factors. ${ }^{1}$ It results from acute ischemia of the optic nerve head in a predisposed patient following a precipitating event. While many of the predisposing factors in this condition are largely undefined, the net effect is to reduce perfusion pressure or increase blood flow resistance in the optic nerve head beyond some critical level ${ }^{2}$. In addition to vascular factors, mechanical factors of the optic nerve head itself, such as a small anomalous optic disc, are thought to predispose certain individuals to NAION. ${ }^{3}$ Also, development and progression of nonarteritic anterior ischemic optic neuropathy is significantly correlated with nocturnal arterial hypotension, particularly in hypertensive patients receiving oral hypertensive therapy. ${ }^{4}$
It is a self limiting disease and there is no effective therapy to treat patients acutely or to prevent recurrence. After 6 months of careful follow-up, more then half of the patients will have no significant change or worsening of their vision in the involved eye Involvement of the opposite eye occurs within 3 years in less than $43 \%$ of patients. ${ }^{1}$

Despite a huge volume of literature that has accumulated on its various aspects over the past 3 decades, information on the natural history of visual outcome is scanty, and when available, it is based on retrospective evaluation, usually of a small number of eyes and often from a mixed group of treated (with corticosteroids) and untreated patients. ${ }^{5}$

Moreover, in a first time study done in Southeast Asia, ${ }^{6}$ the information about incidence in Indian population is less. The discussions regarding visual improvement or deterioration in these studies are contradictory and confusing. There has been only one reported prospective study on the natural history of visual outcome in NAION, which was done as a part of a randomized multicenter trial. ${ }^{7}$ 
Visual deterioration in NAION may be that of visual acuity and/or visual fields. ${ }^{5}$ Non-arteritic anterior ischemic optic neuropathy patients are anxious to know their chances of visual improvement or further deterioration. An attempt to study risk factors, clinical characteristics and natural history of visual outcome in an Indian population can lead us to a perspective into a disease that may be the ocular manifestation of an underlying systemic disorder.

\section{Anatomy of Optic Nerve Head}

The optic nerve head is divided into four parts: ${ }^{8,9}$

Superficial Nerve Fibre Layer: It consists of axonal bundles converging into the optic disc. It is covered by Internal limiting membrane of Elschnig (formed by Muller cells). It is thickened at the centre and called the central meniscus of Kuhnt.

Pre Laminar Portion: It contains bundles of axons lying within astrocytic channels. The axons are loosely bound hence swelling of axons takes place easily (clinically seen as disc oedema). There exists Border Tissue OF Jacoby which separates optic nerve from the choroid. Forward extension of this tissue is called the Intermediary Tissue of Kuhnt. Border tissue of Elschnig is a rim of sclera surrounding the optic nerve.

Laminar Portion: It is a specialized arrangement of glial columns and connective tissue plates that form 200300 holes through which all optic nerve axons pass.

The fenestrations of the lamina cribrosa form a tight seal that shields the relatively high intraocular pressure from the retrolaminar tissues. In cases of increased intracranial pressure, which is transmitted along the sub arachnoid space perineurally can displace the lamina cribrosa anteriorly, which can also damage the neurons. ${ }^{10}$ Largest pores present in superior and inferior parts hence less support in these areas.

The scleral canal in bounded by the border tissue which separates nerve fibres from choroid and sclera. It is $0.5 \mathrm{~mm}$ in length and clinically seen as the scleral ring around optic nerve head.

Retrolaminar Portion: At this point the diameter of the optic nerve doubles due to myelination. Myelination proceeds anteriorly and stops postnatally.

$\mathrm{ONH}$ is a major zone of transition because of:

1. The nerve fibres pass from an area of relatively high intraocular pressure to the lower pressure zone of the retro orbital segment of the optic nerve which is equivalent to the intracranial pressure.

2. The nerve fibres leave the blood supply of the central retinal artery to receive blood from branches of the posterior ciliary and ophthalmic arteries.

3. The nerve fibres make a 90 degree turn and enter the tight confines of the lamina cribrosa.

4. The nerve fibres become myelinated behind the lamina cribrosa.

\section{Aims and Objectives}

Research Question: What are the associated risk factors, common clinical features and visual outcome in cases of NA-AION?

Research Hypothesis: There is a significant improvement in visual acuity over 6 months which corelates with decreasing RNFL thickness on OCT.

Null Hypothesis: There is no co-relation between improving visual acuity and decreasing RNFL thickness on OCT over 6 months

Primary outcome measures were the change in best corrected visual acuity (BCVA) and RNFL thickness on OCT from presentation to 6 months.

Secondary outcome measures of the study was involvement of the other eye during the 6 months duration.

\section{Materials and Methods}

Study Design: This is a prospective, observational, clinical study design.

Study Group: A total of 60 eyes of 60 consecutive adult patients with disc oedema, due to Non Arteritic AION, presenting in the Neuro-ophthalmology clinic of a tertiary care eye hospital, were enrolled over a period of 18 months (in specified period) according to the inclusion criteria.

The study, including patient recruitment and data collection, was approved by the Institutional Review Board of Aravind Eye Hospital. All patients provided written informed consent after they received information about the disease and its relevant investigations along with the risks associated.

Sample collection was done by application of inclusion and exclusion criteria on the patients presenting to the clinic.

\section{Duration of follow up:}

The study was carried out for a period of 6 months for each subject.

\section{Inclusion Criteria}

1. All New Cases of optic disc oedema with history of sudden visual loss, confirmed to have NA-AION on direct ophthalmoscopy or 90D slit lamp biomicroscopy.

2. Cases with optic disc related visual field defects, not due to glaucoma

3. Cases with only background diabetic retinopathy (mild to moderate non proliferative diabetic retinopathy)

\section{Exclusion Criteria}

1. Cases with previous history of NA-AION in fellow eye.

2. Cases with any retinal or optic nerve lesion affecting the visual outcome

3. Cases with diabetic retinopathy, with active neovascularization, vitreous haemorrhages or any complications affecting visual outcome or fields

4. Cases diagnosed to have glaucoma and related field defects 
Each subject underwent a comprehensive neuronophthalmologic examination including:

A. Complete detailed history including:

1. Chief complaints

2. Relevant ocular and systemic history

3. Past history

4. Treatment history

B. Comprehensive examination including:

1. Best Corrected Visual Acuity (BCVA) estimation using Snellen's eye chart and converted into $\log$ MAR visual acuity (logarithm of the minimal angle of resolution) for statistical analysis.

2. Slit lamp evaluation of anterior segment specifically papillary reaction to light.

3. Fundus evaluation by Slit lamp biomicroscopy and indirect ophthalmoscopy.

4. Colour vision by Ishihara pseudoisochromatic charts.

5. Visual field evaluation by Bjerrums tangent screen (for central fields) and Humphrey Field Analysis (HFA) 30-2 programme.

6. Fundus photography \& fundus fluorescein angiography.

7. Cirrus $^{\mathrm{TM}} \mathrm{HD}-\mathrm{OCT}$ version 5.1.1.4: RNFL and ONH analysis report parameters: Based on the $6 \mathrm{~mm} \times 6$ $\mathrm{mm}$ data cube captured by the optic disc cube 200x200 scan, report shows assessment of RNFL and $\mathrm{ONH}$ for both eyes.

8. Blood pressure

9. Blood investigations including:
a. Glycosylated haemoglobin(HbA1c)
b. Serum Random blood sugar (RBS) levels
c. Complete Hemogram
d. Erythrocyte Sedimentation Rate(ESR)
e. C-reactive protein(CRP)
f. Serum Lipid profile

10. Carotid Doppler as indicated

Management was conservative and systemic control of metabolic syndromes was advised for each case as required.

Follow up was advised at 1 month and 6 months from the time of presentation.

Following investigations were repeated at each visit:

1. Visual acuity by Snellens

2. Slit lamp evaluation of anterior segment.

3. Colour vision

4. Visual field evaluation

5. Slit lamp biomicroscopy and indirect ophthalmoscopy

6. Optical coherence tomography

7. Fundus photography

The data was collected and analysed.
Analysis

The study subjects were described and matched with respect to their demographic and clinical characteristics before the analysis, such as age, sex vision, colour vision, and visual field. Their progress from first visit to final follow up was sequentially analysed and interpreted by Student's unpaired ' $t$ ' test for visual acuity in terms of $\log$ MAR scale.

Friedman's coefficient and paired ' $t$ ' test were used to compare RNFL thickness from first to final visit. Statistical analysis was performed using a commercially available statistical software package (STATA, version 11.0, Texas, and USA). All $p$ values were considered statistically significant when the values were less than 0.05 .

\section{Observation and Results Demographics}

Age Distribution of the Subjects: Of the 60 subjects, average age at presentation seen was $59.7 \pm 10$ years. The range was highly variable and was between 39 t0 82 years.

Out of total 60 affected eyes of 60 patients, $71.67 \%$ were males and rest were females showing a male preponderance and a ratio of approx. 2.5:1.

Of the 60 affected eyes right eye showed slight dominance being involved in $56.67 \%$ of cases.

Diabetes mellitus and systemic hypertension were the most widely associated systemic diseases seen, with diabetes mellitus being associated in $25 \%$ and Hypertension seen in $8.33 \%$ of the cases. Combined both of them are seen in $20 \%$ of cases.

Clinical Results: Almost half (43.33\%) of the patients had visual acuity between $6 / 60$ to $1 / 60$ at presentation followed by $38.33 \%$ in the range of $6 / 18$ to $6 / 36$.

Subsequently at 1 month follow up, almost same visual acuity was found in patients with $6 / 18$ to $6 / 36$ at presentation $(36.67 \%)$ suggestive of minimal improvement for this range

Table 1: Visual acuity (Snellens) at 6 months

\begin{tabular}{|l|c|c|}
\hline Visual acuity-6 months & Freq. & Percent \\
\hline $6 / 6-6 / 12$ & 19 & 31.67 \\
\hline $6 / 18-6 / 36$ & 19 & 31.67 \\
\hline $6 / 60-1 / 60$ & 18 & 30 \\
\hline$>1 / 60$ & 4 & 6.67 \\
\hline Total & 60 & 100 \\
\hline
\end{tabular}

At the final visit, $31.67 \%$ patients had visual acuity better than $6 / 12$ whereas $30 \%$ still had visual acuity in range of $6 / 60$ to $1 / 60$.

Visual acuity compared at presentation and first month showed a statistically significant improvement.

Table 2: Visual acuity: comparison at presentation and 6 months follow up

\begin{tabular}{|l|c|c|c|}
\hline \multicolumn{1}{|c|}{ Visual Acuity (LogMAR) } & Mean & SD & P-Value \\
\hline Visual Acuity Presentation & 1.0103 & 0.0906 & $<0.0001$ \\
\hline Visual Acuity 6 Month & 0.682 & 0.7011 & \\
\hline
\end{tabular}


Visual acuity compared at presentation and 6 months also showed a statistically significant improvement A comparison of the mean visual acuity
(LogMAR) at each visit shows significant improvement at each visit

Table 3: Line improvement in Visual acuity by Snellens chart from presentation to 6 months follow up

\begin{tabular}{|l|c|c|}
\hline $\begin{array}{c}\text { Line improvement from presentation to 6 } \\
\text { months }\end{array}$ & Frequency & Percentage \\
\hline 1 Line improvement & 8 & 13.33 \\
\hline$\geq 2$ Line & 24 & 40.00 \\
\hline No Improvement & 28 & 46.67 \\
\hline Total & 60 & 100 \\
\hline
\end{tabular}

Out of 60 patients, 32 patients showed improvement in visual acuity at 6 months. Thus out of total 60 patients, $40 \%$ showed improvement of more than and equal to 2 lines on Snellen's chart. Although $46.7 \%$ of total cases showed no improvement.

In Anterior segment, a Relative Afferent Pupillary Defect was seen in $81.67 \%$ of casess.

The most common and consistent ocular fundus finding was Optic nerve head edema which was seen as the only feature in $50 \%$ of cases.

A significantly small cup in the unaffected eye was seen by fundoscopy in $25 \%$ cases. Colour vision was found to be abnormal in 75\% of cases with rest being normal. The characteristic inferior altitudinal field defect was seen in $40 \%$ of cases and generalized field constriction was seen in $36.67 \%$ of cases.

Table 4: Humphrey field analysis

\begin{tabular}{|l|c|c|}
\hline Field defect & Freq. & Percent \\
\hline $\begin{array}{l}\text { Inferior Altitudinal Field } \\
\text { Defect }\end{array}$ & 36 & 60 \\
\hline Inferior Nasal Field Defect & 7 & 11.67 \\
\hline $\begin{array}{l}\text { Generalized Field } \\
\text { Constriction }\end{array}$ & 9 & 15 \\
\hline Not able to fixate & 7 & 11.67 \\
\hline $\begin{array}{l}\text { Others (Centrocaecal } \\
\text { scotoma) }\end{array}$ & 1 & 1.67 \\
\hline Total & 60 & 100 \\
\hline
\end{tabular}

The inferior altitudinal field defect was seen most commonly, in $60 \%$ of cases followed by inferior nasal field defect seen in $11.67 \%$ cases.

FFA showed the characteristic differential filling on optic nerve head in early phases of angiogram in majority of the cases.

Blood Investigations: Serum C-reactive protein (CRP) and Erythrocyte Sedimentation Rate (ESR) was done in all patients and was found to be normal. A raised serum triglyceride level was seen in $8.33 \%$ patients

\section{Table 5: Serum glycosylated hemoglobin (HbA1c)} levels

\begin{tabular}{|l|c|c|c|}
\hline \multicolumn{2}{|c|}{ HbA1c } & Number & Percentage \\
\hline \multirow{2}{*}{ DM patients } & $>8$ & 10 & 25 \\
\cline { 2 - 4 } & $<8$ & 12 & 30 \\
\hline \multirow{2}{*}{ non DM patients } & $<7$ & 14 & 35 \\
\cline { 2 - 4 } & $>7$ & 4 & 10 \\
\hline \multicolumn{2}{|l|}{ Total } & 40 & 100 \\
\hline
\end{tabular}

40 out of 60 patients underwent HbA1c testing.22 were known cases of DM of which $25 \%$ patients had value greater than $8 \%$. In non diabetic cases, only $10 \%$ had values more than $7 \%$.

\section{Optical Coherence Tomography}

Table 6: OCT: Comparison of average RNFL thickness in affected eye at presentation, 1 month and 6 months follow up - Friedman test

\begin{tabular}{|l|c|c|c|c|c|c|c|c|c|}
\hline Quadrant & \multirow{2}{*}{ No. } & \multicolumn{2}{|c|}{ 1 st visit } & \multicolumn{2}{c|}{$\mathbf{2}^{\text {nd }}$ visit } & \multicolumn{2}{c|}{ 3rd visit } & Friedman & \multirow{2}{*}{ Significance } \\
& & & Mean & S.D & Mean & S.D & Mean & S.D & \\
\hline coeficient & \\
\hline Naserior & 60 & 288.7 & 68.596 & 125.2 & 7.78 & 90 & 8.25 & 120 & $\mathrm{p}<0.0001$ \\
\hline Inferior & 60 & 184.58 & 60.908 & 98.33 & 12.19 & 68.91 & 8.55 & 109.766 & $\mathrm{p}<0.0001$ \\
\hline Temporal & 60 & 258.6 & 91.505 & 137.23 & 12.79 & 104.3 & 11.04 & 116.033 & $\mathrm{p}<0.0001$ \\
\hline Average & 60 & 77.416 & 19.199 & 63.03 & 10.18 & 48.36 & 7.22 & 88.233 & $\mathrm{p}<0.0001$ \\
\hline
\end{tabular}

A trend of decreasing RNFL thickness (in microns) is seen in affected eye from presentation to the last visit at 6 months follow up which is statistically significant.

\section{Discussion}

The main objective of our study is to analyze the risk factors, clinical features and visual outcome in cases of NA-AION (non arteritic anterior ischemic optic neuropathy). 
There are very few studies in the past which compare and rationalise all the three aspects i.e. risk factors, clinical features and visual outcome, almost none in an Indian population.

In our study a total of 60 eyes of 60 patients newly diagnosed as cases of non-arteritic AION, and confirmed by clinical evaluation, were followed up each over 6 months. The study was done over a period of two years.

The study data was analysed demographically and statistically.

In our study $71.67 \%$ patients were males, mean age being $59.7 \pm 10$ years.

Tian GH et al, ${ }^{11}$ demonstrated the age of onset in NAION is relatively early $50.90+/-8.88$ years (range 34 - 78 years) and the median is 50 years and males seem to have a high incidence.

The most common risk factor is metabolic syndromes i.e. combination of medical disorders that, when occurring together, increase the risk of developing cardiovascular disease and diabetes.

In determining the risk factors, diabetes mellitus seems to be the most associated risk factor, present in $25 \%$ cases (second being systemic hypertension in $8.3 \%$ patients) for the development of NA-AION. There were no known risk factors in $25 \%$ cases who subsequently underwent a thorough systemic evaluation.

In a similar study done by Daniel A Jacobson et al, ${ }^{12}$ Diabetes mellitus seems to be a major risk factor for the development of nonarteritic anterior ischemic optic neuropathy.

Among ocular risk factors, a small cup was seen in other eye by slit lamp biomicroscopy in $25 \%$ cases.

S S Hayreh, ${ }^{13}$ also showed a significant association of a small cup i.e. 'disk at risk' in cases on NA-AION

Colour vision was abnormal in $75 \%$ of cases and Central fields showed the field defects (generalized field constriction and inferior altitudinal defect) in $60 \%$ cases.

The Humphrey field analysis showed the characteristic Inferior altitudinal field defect in $60 \%$ of cases and an inferior nasal defect in $11.6 \%$ cases.

S S Hayreh et al, ${ }^{14}$ demonstrated in the past that an absolute inferior nasal visual field defect is much more common $(22.4 \%)$ than an absolute inferior altitudinal visual field defect (8.0\%) in NA-AION and could be considered the most characteristic single field defect in NA-AION.

Also, he found that a combination of relative inferior altitudinal defect with absolute inferior nasal defect is usually the most common pattern in NA-AION.

In fluorescein angiograms, FFA (fundus fluorescein angiography) was recorded for all the cases and showed the typical differential filling on the optic nerve head in early pictures (The normal part of the optic disc filled normally while the ischemic part either did not fill or filled very late during the transit of the dye depending upon the age of the lesion).

Same conclusions were also noted down by $\mathrm{S} S$ Hayreh et al, ${ }^{15}$ which also cited that the angiographic pattern varied not only with the degree of involvement of the optic disc but also with the interval between the onset of AION and the angiographic examination.

OCT findings in the study showed that there was an overall increase in the RNFL thickness in the affected eyes at presentation. However, with time the average RNFL thickness decreased significantly. Average RNFL thickness: $202.3 \pm 56.1$ microns at presentation as compared to $77.9 \pm 5.1$ microns at 6 months follow-up visit.

Similar findings were noted in a study by Giacomo Savini,${ }^{16}$ Costantino Bellusci et al, ${ }^{17}$ who concluded that OCT (stratus) can effectively quantify RNFL thickness in cases with disc oedema due to AION, ON and IIH and can be of use in learning the pathophysiology of the condition.

The most important piece of information required in NAION, from the point of view of both patient and ophthalmologist, is the natural history of visual outcome. $^{5}$

The change in visual acuity was inversely related with the RNFL thickness.

There have been similar studies in the past correlating the visual function change with the RNFL thickness values in cases with disc oedema. Ines Contreras et $a l,{ }^{18}$ in their study of RNFL thickness in eyes with NAION concluded that OCT gives an objective outcome measure which correlates well with visual function.

In our study there was improvement in visual acuity over 6 months follow up in $53.3 \%$ cases which is statistically significant and correlates well with decreasing RNFL oedema.

However as pointed by S S Hayreh et al ${ }^{14}$ it is established that visual acuity gives information basically about the functioning of only the fovea and the papillomacular nerve fibres in the optic nerve, and not of the entire retina or the entire optic nerve. Nonarteritic anterior ischemic optic neuropathy may involve the entire optic nerve head or only one part of it; in some cases, the papillomacular nerve fibres may not be involved at all, which explains the presence of normal visual acuity in many eyes with NAION.

In our study patients were followed up with both visual acuity (Snellen's) and Visual Fields (HFA) which provide very useful information about the visual status of the eye, also the changes in two can be totally unrelated and independent to each other.

The incidence of other eye involvement was 3.3\%, as patients were followed up for short time of 6 months in our study.

According to Niraj Desai et al, ${ }^{1}$ involvement of the opposite eye occurs within 3 years in less than $43 \%$ of patients.

The limitation in our study of natural history of visual outcome in NAION is that it could not document the visual function from its day of onset of NAION onward. Our visual evaluation can only be from the time 
the patients were first seen in our clinic and not from the date of onset of visual loss in NAION, so that if there was deterioration or improvement before we saw the patient, that information is not available.

Also, due to a close follow up of 6 months the long term trend of visual progression and stabilization could not be documented.

\section{Summary}

1. This is a prospective, observational, clinical study design done to evaluate the various clinical parameters pertaining to NA-AION in a South Indian population

2. A total of 60 eyes of 60 patients were studied.

3 . These eyes were studied at regular intervals over a total period of 6 months each.

4. At each visit they were evaluated for clinical markers of optic nerve function like, visual acuity, FFA, colour vision and visual fields by HFA. OCT was used to record the peripapillary RNFL thickness of each quadrant in the affected and the unaffected eyes, at each visit.

\section{The major outcomes of the study were:}

1. Metabolic Syndromes are the most significantly associated risk factors for the occurrence of NA AION.

2. Clinical investigations mainly show the characteristic findings as mentioned in literature, with very little variation. However the importance of clinical examination, mainly pupillary reaction and fundus evaluation by slit lamp biomicroscopy, cannot be overlooked.

3. Visual acuity is highly variable at presentation with cases even presenting with normal or near normal visual acuity. Visual outcome showed significantly improvement within 6 months of presentation.

\section{Conclusion}

Non arteritic anterior ischemic optic neuropathy (NAION) was the most common form of ischemic optic neuropathy in our study.

It is an idiopathic, ischemic insult of the optic nerve head characterized by acute, monocular, painless visual loss with optic disc swelling.

The systemic risk factor which we found to be most strongly associated with was diabetes mellitus followed by systemic hypertension. Also an ocular risk factor of small cup in the other eye was observed.

Visual acuity at presentation was varied in a large range. Some cases however presented with normal or near normal visual acuity at the first visit itself.

For patient evaluation, all the investigations showed the classical description of the disease, as given in literature.

OCT showed increase in RNFL thickness (overall and sectoral in few) at presentation with a progressive decrease and stabilization over 6 months time.
HFA also showed the characteristic "inferior altitudinal field defect" in majority of cases.

In visual outcome, visual acuity showed improvement over a 6 months follow up period in more than half of the cases $(53.3 \%)$ though visual fields had persistent field defect.

We have few inherent limitations in the study mainly with the occurrence of symptoms and time of presentation of the patient, which may have a bearing on the final outcome of the disease. Also a short follow up limited us regarding study of the stabilization of visual acuity over a longer duration.

However our study gives an overview of prevalent risk factors and clinical characteristics. It also shows a significant visual outcome after 6 months in cases of NA-AION.

\section{References}

1. Desai N. Nonarteritic Anterior Ischemic Optic Neuropathy. J Clin Hypertens. 2005;7:130-133.

2. Hayreh SS. Acute ischemic disorders of the optic nerve: pathogenesis, clinical manifestations and management. Ophthalmol Clin North Am. 1996;9:407-42.

3. Beck RW, Servais GE, Hayreh SS. Anterior ischemic optic neuropathy: IX. Cup-to-disc ratio and its role in pathogenesis. Ophthalmology. 1987;94(11):1503-8.

4. Hayreh SS. Role of nocturnal arterial hypotension in the development of ocular manifestations of systemic arterial hypertension. Current Opinion in Ophthalmology. 1999;10(6):474-82.

5. Hayreh SS, Zimmerman MB. Nonarteritic anterior ischemic optic neuropathy: natural history of visual outcome. Ophthalmology. 2008;115(2):298-305.

6. Cullen JF. Optic neuritis in Southeast Asia: a different pattern of disease. Asian Journal of Ophthalmology. 2011;12(4):216-7.

7. Kaufman D, Dickersin K, Kelman S, Langenberg P, Newman N, Wilson PD. Ischemic optic neuropathy decompression trial: twenty-four-month update. Archives of Ophthalmology. 2000;118(6):793-8.

8. Wolff E. Orbit and eyelids. Wolff's Anatomy of the Eye and Orbit Eighth edition. 1997:489-536.

9. Joseph F. Embryology, Anatomy and Physiology of Afferent visual pathway. Walsh and Hoyt's Clinical Neuro Ophthalmology Sixth edition. 2004:24-35.

10. Morgan WH, Yu DY, Cooper RL, Alder VA, Cringle SJ, Constable IJ. The influence of cerebrospinal fluid pressure on the lamina cribrosa tissue pressure gradient. Investigative Ophthalmology \& Visual Science. 1995;36(6):1163-72.

11. Tian GH, Jia N, Lu CF. Clinical characteristics of nonarteritic anterior ischemic optic neuropathy. 2009;45(12):1064-7.

12. McCulley TJ, Lam BL, Feuer WJ. Incidence of nonarteritic anterior ischemic optic neuropathy associated with cataract extraction 1 . Ophthalmology. 2001;108(7):1275-8.

13. Hayreh SS. Ischemic optic neuropathy. Progress in retinal and eye research. 2009;28(1):34-62.

14. Kupersmith MJ, Frohman L, Sanderson M, Jacobs J, Hirschfeld J, Ku C, Warren FA. Aspirin reduces the incidence of second eye NAION: a retrospective study. Journal of neuro-ophthalmology: the official journal of the North American Neuro-Ophthalmology Society. 1997;17(4):250-3. 
15. Hayreh SS. Anterior ischaemic optic neuropathy. II. Fundus on ophthalmoscopy and fluorescein angiography. The British Journal of Ophthalmology. 1974;58(12):964.

16. Giacomo Savini. Detection and quantification of retinal nerve fiber layer thickness in optic disc oedema using stratus. Arch Ophthal. 2006;124:1111-1117.

17. Bellusci C, Savini G, Carbonelli M, Carelli V, Sadun AA, Barboni P. Retinal nerve fiber layer thickness in nonarteritic anterior ischemic optic neuropathy: OCT characterization of the acute and resolving phases. Graefe's Archive for Clinical and Experimental Ophthalmology. 2008;246(5):641-7.

18. Anderson DR, Patella VM. Automated Static Perimetry. 2nd edition, St Louis, Mosby, 1998.

How to cite this article: Sharma A.V, Sharma A. Analysis of risk factors, clinical features and visual outcome in cases of non-arteritic anterior ischemic optic neuropathy (NA-AION) at a tertiary eye care centre in India. Indian J Clin Exp Ophthalmol. 2018;4(4):541-547. 